\title{
Dynamical properties of the soft-wall elliptical billiard
}

Tiago Kroetz ${ }^{\mathrm{a}, *}$, Hércules A. Oliveira ${ }^{\mathrm{b}}$, Jefferson S. E. Portela ${ }^{\mathrm{a}}$, Ricardo L. Viana $^{\mathrm{c}}$

${ }^{a}$ Universidade Tecnológica Federal do Paraná, Pato Branco, Paraná, Brazil

${ }^{b}$ Universidade Tecnológica Federal do Paraná, Ponta Grossa, Paraná, Brazil

${ }^{c}$ Universidade Federal do Paraná, Curitiba, Paraná, Brazil

\begin{abstract}
Physical systems such as optical traps and microwave cavities are realistically modeled by billiards with soft walls. In order to investigate the influence of the wall softness on the billiard dynamics, we study numerically a smooth two-dimensional potential well that has the elliptical (hard-wall) billiard as a limiting case. Considering two parameters - the eccentricity of the elliptical equipotential curves and the wall hardness, which defines the steepness of the well - we show that (i) whereas the hard-wall limit is integrable and thus completely regular, the soft wall elliptical billiard exhibits chaos, (ii) the chaotic fraction of the phase space depends non-monotonically on the hardness of the wall, and (iii) the effect of the hardness on the dynamics depends strongly on the eccentricity of the billiard.
\end{abstract}

Keywords: soft-wall billiard, elliptical billiard, Hamiltonian system, parameter space

PACS: 05.45.-a, 05.45.Ac

\section{Introduction}

A billiard is a system where a particle moves freely in a region enclosed by a boundary with which the particle suffers elastic collisions. The dynamics in such systems can be very rich and is completely determined by the geometry

\footnotetext{
*Corresponding author

Email addresses: kroetz@utfpr.edu.br (Tiago Kroetz), hercules@utfpr.edu.br (Hércules A. Oliveira), stafusa@utfpr.edu.br (Jefferson S. E. Portela), viana@fisica.ufpr.br (Ricardo L. Viana)
} 
of the boundary [1]. Billiards have found several applications in physics, proving themselves particularly useful for the study of quantum chaos and its relation to classical nonlinear phenomena $[2,3,4,5,6,7,8]$.

The elliptical billiard presents only regular motion, as constants of motion restrict the orbits to invariant curves in phase space [9]. These integrable orbits come in two types: the libration (always passing between the foci) and the rotation (never passing between the foci). The regions in phase space that contain each type of orbit are separated by the so-called separatrix.

This separation of two distinct kinds of motion motivated the study of the escape rates of an elliptical billiard with a hole placed on its boundary, considering billiards with both static and time-dependent walls [10]. The time-dependent elliptical billiard also permitted the investigation of Fermi acceleration, where particles can exhibit unlimited energy growth by impacting with the moving walls $[11,12,13,14]$. Since the dynamics in a static elliptical billiard is integrable, the existence of Fermi acceleration in the time-dependent elliptical billiard effectively extends the scope of the LRAConjecture [15]. This conjecture states that static billiards presenting chaotic dynamics will display Fermi acceleration whenever their non-static counterpart boundaries have a periodic dependence with time. A separatrix is an homoclinic or an heteroclinic surface in phase space which is very sensitive to small perturbations, easily replaced by an stochastic layer due to a resonant interaction phenomena $[16,17]$. Due this fact, even billiards with integrable dynamics but with a separatrix in phase space exhibit Fermi acceleration when a time-dependence is imposed to the boundaries.

Besides time-dependent walls, another possible generalization of the concept of billiard is that of a soft-wall system, where the Heaviside step potential that corresponds to the rigid wall of traditional, hard billiards is replaced by a smooth potential, i. e., the wall is softened. Soft billiard walls provide a more realistic model for trapped particles (e.g., [18]), since physically realizable potentials are typically smooth. Wall softness has so far been observed to have an stabilizing effect on the dynamics. For instance, the emergence of islands of stability from the tangent and corner periodic orbits of dispersing billiards as their walls become soft was shown by Rom-Kedar and Turaev $[19,20,21]$. Cold atoms confined in optical traps have been investigated numerical and experimentally $[22,23,24]$ and soft walls were found to give rise to stable regions in phase space. These results corroborate previous theoretical results for dispersing chaotic billiards and also indicate they might generalize to focusing chaotic billiards. It has already been suggested that 
the softness of the wall could be a tunable parameter to control chaos [24], and indeed, one could ask if wall softness always enhances the stability of a system.

In this work we investigate a realization of the elliptical billiard with soft walls considering a two-dimensional smooth potential as a model $[24,25,26$, 19]. We intend to determine the effect of softness on the dynamics of a billiard that is both integrable at the hard-wall limit and also presents a heteroclinic structure in phase space. We find that, similarly to the generalization of the LRA Conjecture, here too the phase space fragility seems to give rise to unexpectedly rich, unstable behavior.

The paper is organized as follows. In Section 2 we present the elliptical billiard potential, its equations of motion, and typical trajectories. In Section 3 we define a Poincaré surface of section, and investigate the transitions in the phase space as we vary the hardness of the billiard walls. In Section 4 we present the parameter space of the system revealing its non-trivial orderchaos transitions. Finally, we draw our conclusions and final considerations in Section 5

\section{Soft-wall Billiard}

The motion of a particle in a soft-wall elliptical billiard is governed by the two-dimensional Hamiltonian

$$
H=\frac{p_{x}^{2}}{2}+\frac{p_{y}^{2}}{2}+V(x, y)
$$

where $(x, y)$ is the particle position in rectangular coordinates, $p_{x, y}$ are the corresponding momenta, and the potential $V(x, y)$ defines the force exerted on the particle, i. e., the shape of the billiard. In order to obtain a tunable softness and the desirable geometry of the billiard, we choose

$$
V(x, y)=\operatorname{erf}\left[h\left(x^{2}+\frac{y^{2}}{b^{2}}-1\right)\right]
$$

where $\operatorname{erf}(z)=2 \pi^{-1 / 2} \int_{0}^{z} e^{-\tau^{2}} d \tau$ is the error function. The parameter $h$ controls the hardness of the billiard - higher values correspond to steeper walls; and the equipotential curves are ellipses centered at the origin, with eccentricity $e=\sqrt{1-b^{2}}$. The potential $V$ is null over an ellipse with semimajor axis equals to 1 and semi-minor axis equals to $b, V$ is negative in the 
interior of this curve, and positive outside. The hard-wall billiard is recovered as $h \mapsto \infty$, when $V \mapsto 1(-1)$ outside (inside) the null equipotential curve. The dependence of $V$ on the hardness $h$ is illustrated in Fig. 1.
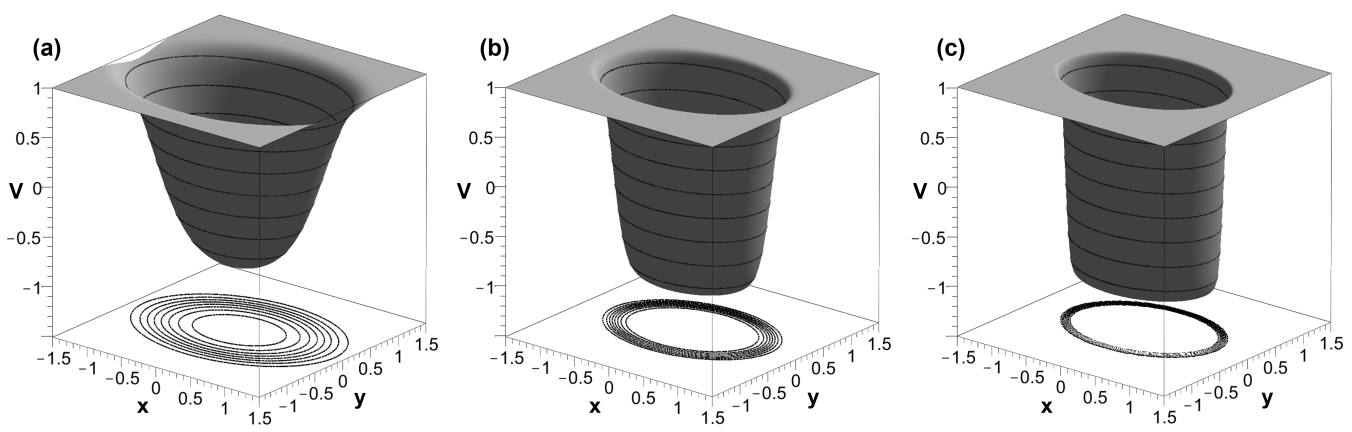

Figure 1: Potential $V(x, y)$ for $e=0.72$ and hardness (a) $h=1$, (b) $h=3$, and (c) $h=8$.

The time evolution of the system is determined by Hamilton's equations

$$
\begin{aligned}
\frac{d x}{d t} & =\frac{\partial H}{\partial p_{x}}=p_{x}, \\
\frac{d p_{x}}{d t} & =-\frac{\partial H}{\partial x}=\frac{-4 h x}{\sqrt{\pi}} \exp \left[-h^{2}\left(x^{2}+\frac{y^{2}}{b^{2}}-1\right)^{2}\right], \\
\frac{d y}{d t} & =\frac{\partial H}{\partial p_{y}}=p_{y}, \\
\frac{d p_{y}}{d t} & =-\frac{\partial H}{\partial p_{y}}=\frac{-4 h y}{b^{2} \sqrt{\pi}} \exp \left[-h^{2}\left(x^{2}+\frac{y^{2}}{b^{2}}-1\right)^{2}\right],
\end{aligned}
$$

which must be solved numerically. We use the Runge-Kutta method with adaptive step size $d t$ and choose $d t=\epsilon_{0}+\epsilon_{1}|V(x, y)|$, with $\epsilon_{0}=10^{-5}$ and $\epsilon_{1}=10^{-3}$, so that the step has an upper limit of order $\epsilon_{1}$ and takes the smaller $\epsilon_{0}$ value where the potential varies more abruptly, around $V(x, y)=0$.

Typical trajectories lying on the energy surface $E=0$ obtained from integrating Eq. 3 can be seen in Fig. 2 and Fig. 3, where the two different initial conditions considered for each figure correspond to a librator orbit and a rotator orbit, respectively, at the hard-wall limit. These figures show how the Lissajous-like orbits of the bow-shaped potential well change into the well-known regular orbits of the hard-wall elliptical billiard $[9,10]$ as the 
hardness $h$ increases. Remarkably, for $h=2.5$, the system exhibits chaotic behavior [Fig. 2(c), 3(c)].
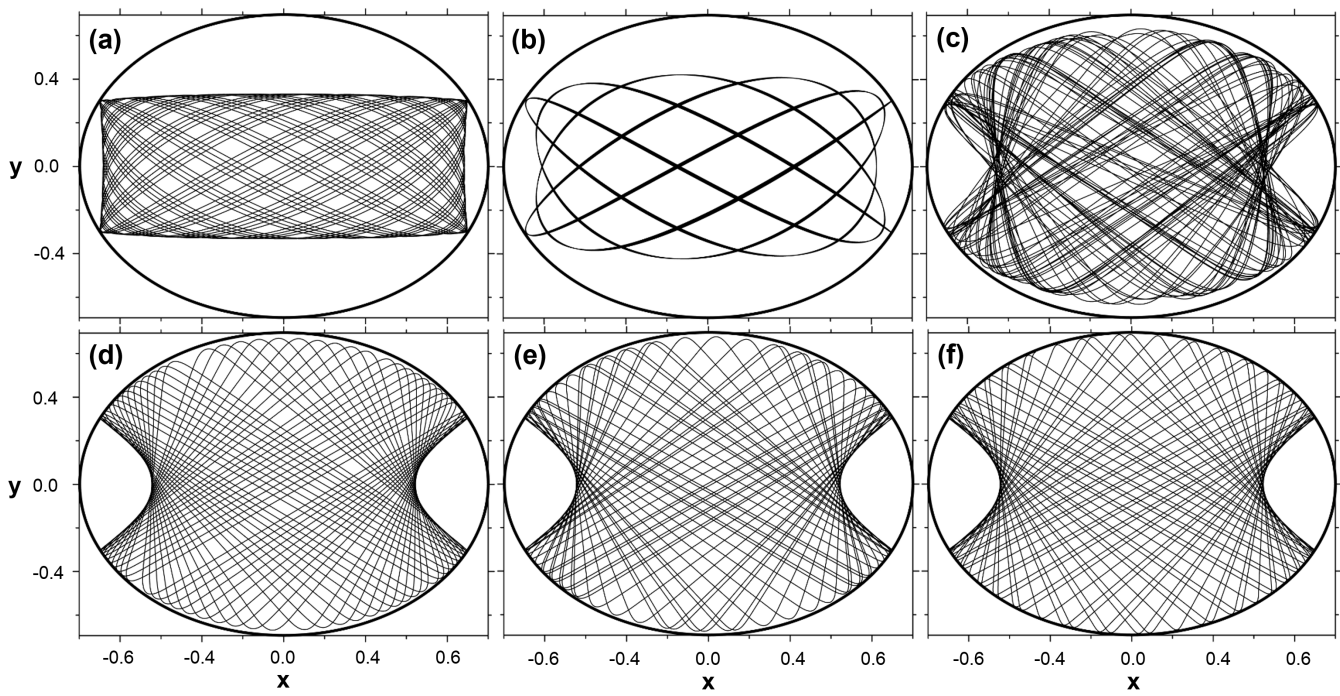

Figure 2: Trajectories of energy $E=0$ for the soft-wall elliptical billiard at several hardness values. The eccentricity is $e=0.72$ and the initial condition is $(x, y)=(0.9,0.3025)$, $\left(p_{x}, p_{y}\right)=(0,0)$. The hardness is (a) $h=0.5$, (b) $h=1$, (c) $h=2.5$, (d) $h=6$, (e) $h=8$, and (f) $h=18$. Also displayed is the equipotential curve $V(x, y)=0$, an absolute "wall" for the particles with the chosen energy.

\section{Poincaré Map}

In conservative systems, the energy is an integral of motion that reduces the effective dimension of the system by one unity. Specifically, adopting polar coordinates $(r, \theta)$ centered at the bottom of the potential well to describe the particle position in the elliptical billiard, $r$ can be shown to be a dependent variable, given by

$$
r=\sqrt{\frac{b^{2}}{b^{2} \cos ^{2} \theta+\sin ^{2} \theta}\left[\frac{h-\operatorname{erf}^{-1}\left(p^{2} / 2-E\right)}{h}\right]} .
$$

An additional dynamical variable can be eliminated from the system description by using a Poincaré surface of section [30], allowing us to describe the 

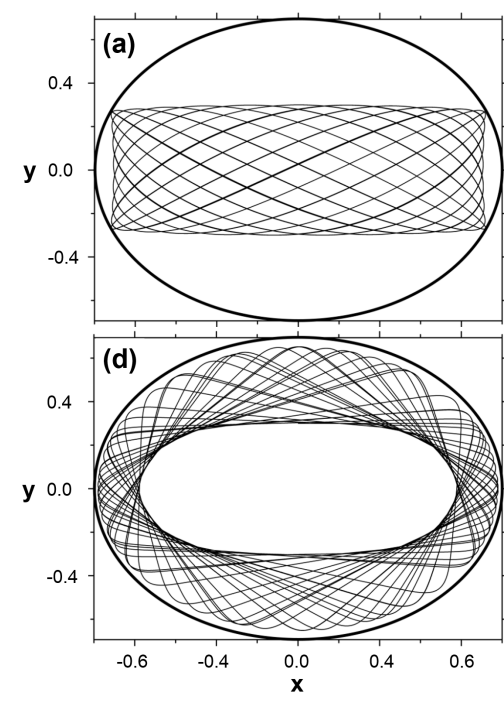
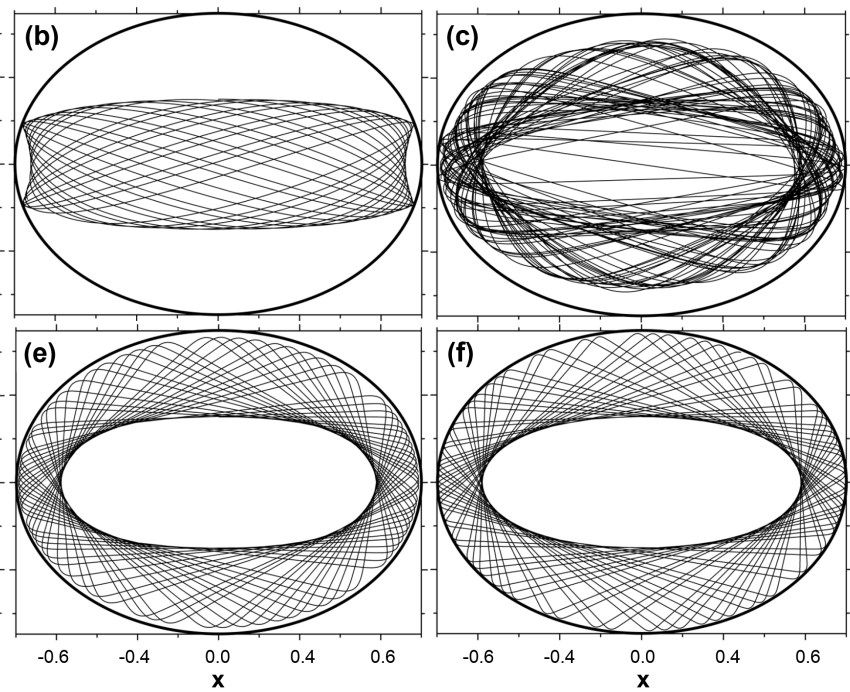

Figure 3: The same as in Fig. 2, with the initial condition $(x, y)=(0,0.3), p_{y}=0$ and $p_{x}$ determined by imposing $E=0$. Hardness values are (a) $h=0.5$, (b) $h=1$, (c) $h=2.5$, (d) $h=6$, (e) $h=8$, and (d) $h=18$, with $p_{x}$ varying between 0.932 and $\sqrt{2}$.

dynamics of the soft-wall elliptical billiard by means of a two-dimensional map. We devote Subsection 3.1 to describe our surface of section and the map phase-space variables. The corresponding numerical results are in Subsection 3.2

\subsection{Poincaré Surface of Section}

Poincaré surfaces for hard-wall billiards are typically defined by reflections with the boundaries, while for smooth dynamical systems the surfaces are usually defined $[27,28,29]$ by a condition such as $x=0$. Although it would also be reasonable to define the Poincaré surface on $x=0$ for the soft-wall elliptical billiard, displaying our results in a similar phase space facilitates comparisons to the well-known properties of the hard-wall elliptical billiard. Therefore we need to define a Poincaré surface which is analogous to the reflections in hard-wall billiards. Reflections are characterized by reversal of the perpendicular component of the momentum relative to the wall, $p_{\perp} \mapsto-p_{\perp}$, a reversal that in soft-wall billiards happens when $p_{\perp}=0$. Thus, we consider that a reflection takes place when the trajectory is tangent to an equipotential curve, as illustrated in Fig. 4. The condition for such a 
reflection can be written, from Eq. 3, as

$$
\vec{p} \cdot \vec{F}=x p_{x}+\frac{y}{b^{2}} p_{y}=0 .
$$

We define the Poincaré surface as the plane $p_{\perp}=0$, which is pierced by a trajectory when the condition above is satisfied, as the dot product changes signal from positive to negative. This corresponds to a reflected trajectory in configuration space $(x, y)$ and to a point in the map phase space $\left(\theta, p_{\|}\right)$, where $p_{\|}$is the component of the momentum parallel to the equipotential ellipse at the reflection and can be normalized with respect to its maximum value

$$
p_{\max }=\sqrt{2[E+\operatorname{erf}(h)]} .
$$

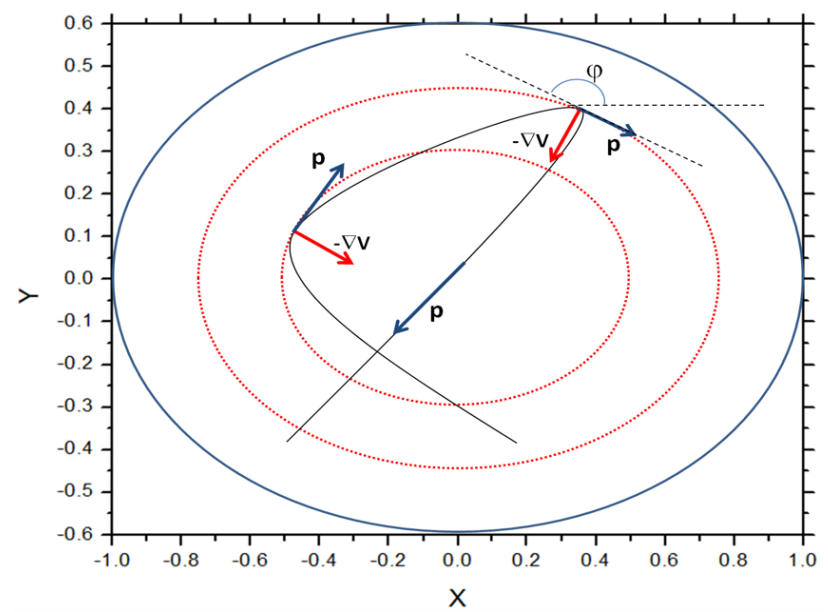

Figure 4: Schematic representation of a trajectory traced by a particle in the soft-wall elliptical billiard. The trajectory crosses the Poincaré surface at each reflection at (tangency to) an equipotential curve. The angle with the horizontal direction of the equipotential curve at each reflection is $\varphi$.

The numerical simulations were performed using rectangular dynamical variables $\left(x, y, p_{x}, p_{y}\right)$. The transformation between these variables at the reflection $\left(p_{\perp}=0\right)$ is given by

$$
\begin{array}{ll}
x=r \cos \theta, & y=r \sin \theta, \\
p_{x}=p_{\|} \cos \varphi, & p_{y}=p_{\|} \sin \varphi,
\end{array}
$$


where $\varphi$ is the angle between the equipotential (and therefore $p_{\|}$) and the horizontal direction, as illustrated in Fig. 4, and is given by

$$
\varphi=\tan ^{-1}\left[\frac{-b^{\prime} x}{a^{\prime 2} \sqrt{1-x^{2} / a^{\prime 2}}}\right],
$$

where $b^{\prime}=\sqrt{x^{2}\left(1-e^{2}\right)+y^{2}}$ and $a^{\prime}=b^{\prime} / \sqrt{\left(1-e^{2}\right)}$ are respectively the semi-minor axis and the semi-major axis of the equipotential ellipse.

\subsection{Influence of Hardness on the Dynamics}

Much of the research on billiards aims to determine the influence of the control parameters on the dynamics of the system, (e.g., [31, 32, 33, 34]). In this subsection, we focus on the transition between integrable and chaotic regimes in the soft-wall elliptical billiard as the hardness is varied. We begin this investigation by analysing Poincaré plots for various values of hardness $h$. In each plot 160 initial conditions uniformly distributed over the phase space are integrated for 5000 reflections. We show in Fig. 5 some representative Poincaré plots for eccentricity $e=0.72$ and energy $E=0$. Empty regions at high $\left|p_{\|}\right|$values indicate that the dynamics does not present reflections close to the direction tangent to the equipotential curves.

Some important features of the elliptical billiard with very soft, bowlshaped walls ( $h=1$, see Fig. 1 (a)) are revealed in Fig. 5 (a) i) absence of chaos; ii) absence of integrable spanning surfaces along $\theta$ direction in the phase space, i.e., rotator orbits as those observed in the hard-wall billiard; and iii) the stability of the orbit over the semi-major axis of the ellipse. The last mentioned orbit corresponds to the points $\left(\theta, p_{\|}\right)=(0,0)$ and $(\pi, 0)$ in the Poincaré plot, which is unstable for the hard-wall case. The islands of quasi-periodicity around $\left(\theta, p_{\|}\right)=(0,0)$ and $(\pi, 0)$ correspond to a set of librator orbits similar to those shown in Fig 2 (b).

It is possible to observe in Fig. 5 (b) that by considering a higher value for the hardness parameter, $h=1.7$, the trajectory over the semi-major axis rapidly loses its stability and bifurcates generating chaotic orbits close to the now unstable point. The two big islands remaining in phase space located around $\left(\theta, p_{\|}\right)=\left(\frac{\pi}{2}, 0\right)$ and $\left(\frac{3 \pi}{2}, 0\right)$ correspond to the librator orbits close to the straight line trajectory over the semi-minor axis, which are also stable for the hard-wall elliptical billiard. Increasing further the hardness, the area occupied by chaotic orbits in the phase space expands and constitute 


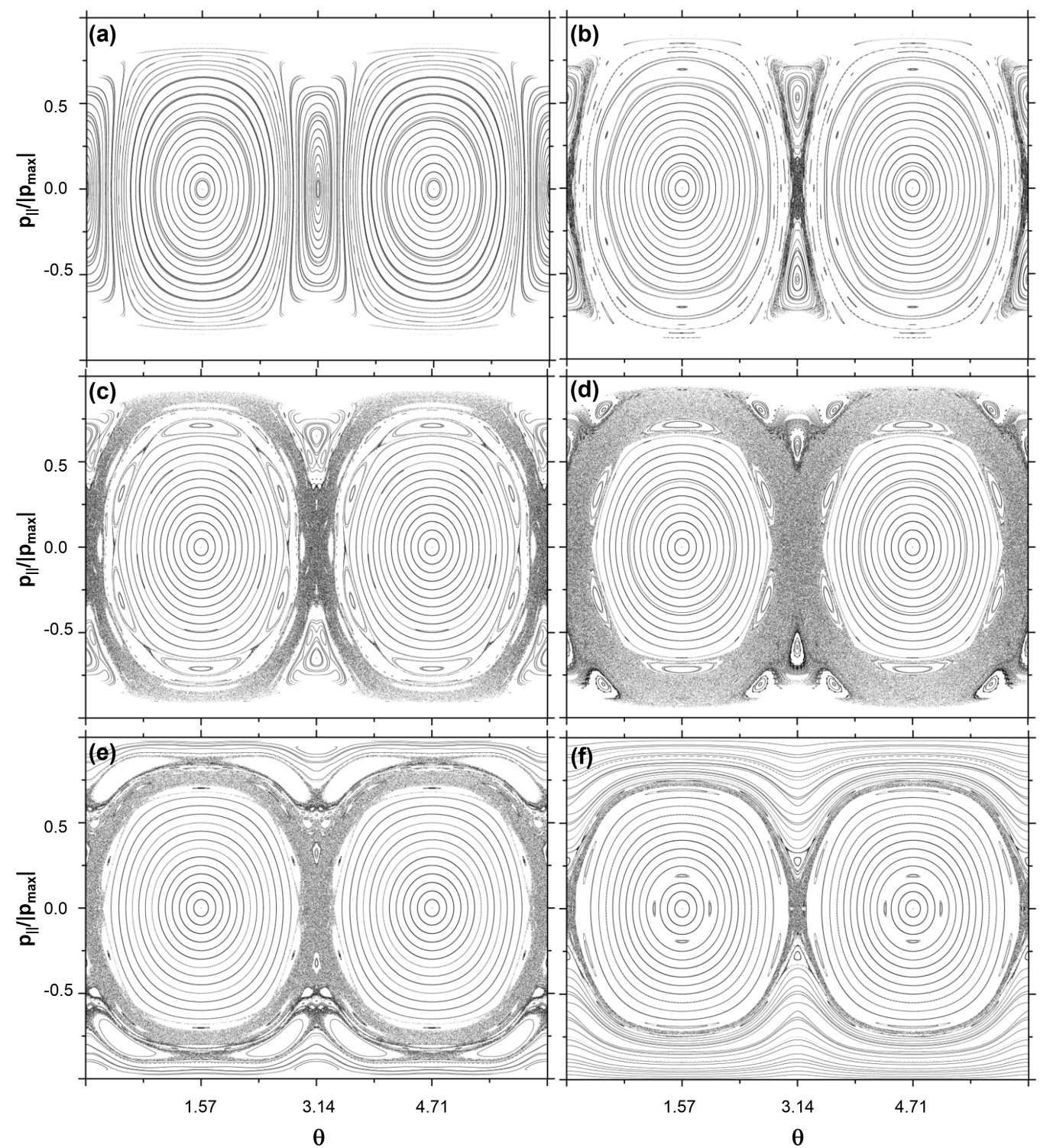

Figure 5: Poincaré plots for the soft-wall elliptical billiard with eccentricity $e=0.72$, energy $E=0$ and hardness (a) $h=1$, (b) $h=1.7$, (c) $h=2$, (d) $h=2.5$, (e) $h=6$ and (f) $h=18$.

a stochastic layer around the islands of librator orbits, as can be seen in Fig. 5 $(\mathrm{c}, \mathrm{d})$. 
Remarkably, the stochastic layer reverts its tendency of growth with $h$ and starts to shrink at around $h=6$. The integrable spanning curves at the top and at the bottom of Fig. 5 (e), for $h=6$, correspond to the rotator orbits around the two foci. In Fig. 5 (f), it is possible to see that for an even higher hardness, for $h=18$, the integrable spanning curves correspondent to the rotator orbits invade the stochastic layer, significantly reducing the presence of chaotic dynamics. At the limit $h \rightarrow \infty$, the hard-wall elliptical billiard is expected to be recovered. In this case, the stochastic layer must vanish and is replaced by a separatrix that segregates the only possible types of orbits in phase space: librations close the semi-minor axis and rotations around the two foci.

This general scenario holds over a wide range of eccentricities. We illustrate this in Fig. 6, with Poincaré plots for the same values of parameter $h$ and energy $E$ as in Fig. 5, but for a higher eccentricity $e=0.84$. Although we also observe an increase followed by a reduction of stochastic layer for $e=0.84$, the area occupied by chaotic orbits is slightly greater when we compare Fig. 5 (d, e) to Fig. 6 (d,e).

The variety of behaviors exhibited by the soft-wall elliptical billiard is illustrated by the configuration space trajectories of Fig. 7, whose initial conditions are shown in the phase space portraits of Fig. 6 . The black square on the left side island on Fig. 6 (a) originates the librator orbit close to the semi-minor axis of Fig. 7 (a). The initial condition marked by a green square on the center island of the same figure leads to a librator orbit close to the semi-major axis of Fig. 7 (b) - a type of librator orbit that is only possible for low values of $h$. The larger libration orbit shown in Fig. 7 (c) corresponds to the initial condition marked by the red square on the outer curve of the island at the right side of Fig. 6 (a). The distinctive trajectory of Fig. 7 (d) corresponds to the initial condition marked by a black square on the small island of Fig. 6 (d). Such satellite islands persist with the changes in parameter $h$ and the corresponding trajectories are typical of soft-wall elliptical billiards. An initial condition in the stochastic layer, as indicated by the red square on the right hand side of Fig. 6 (d), originates a clearly irregular orbit and an initial condition over an integrable spanning curve, as that marked by the black square in Fig. 6 (f), results in the rotator orbit around two foci depicted in Fig. 7 (f). 


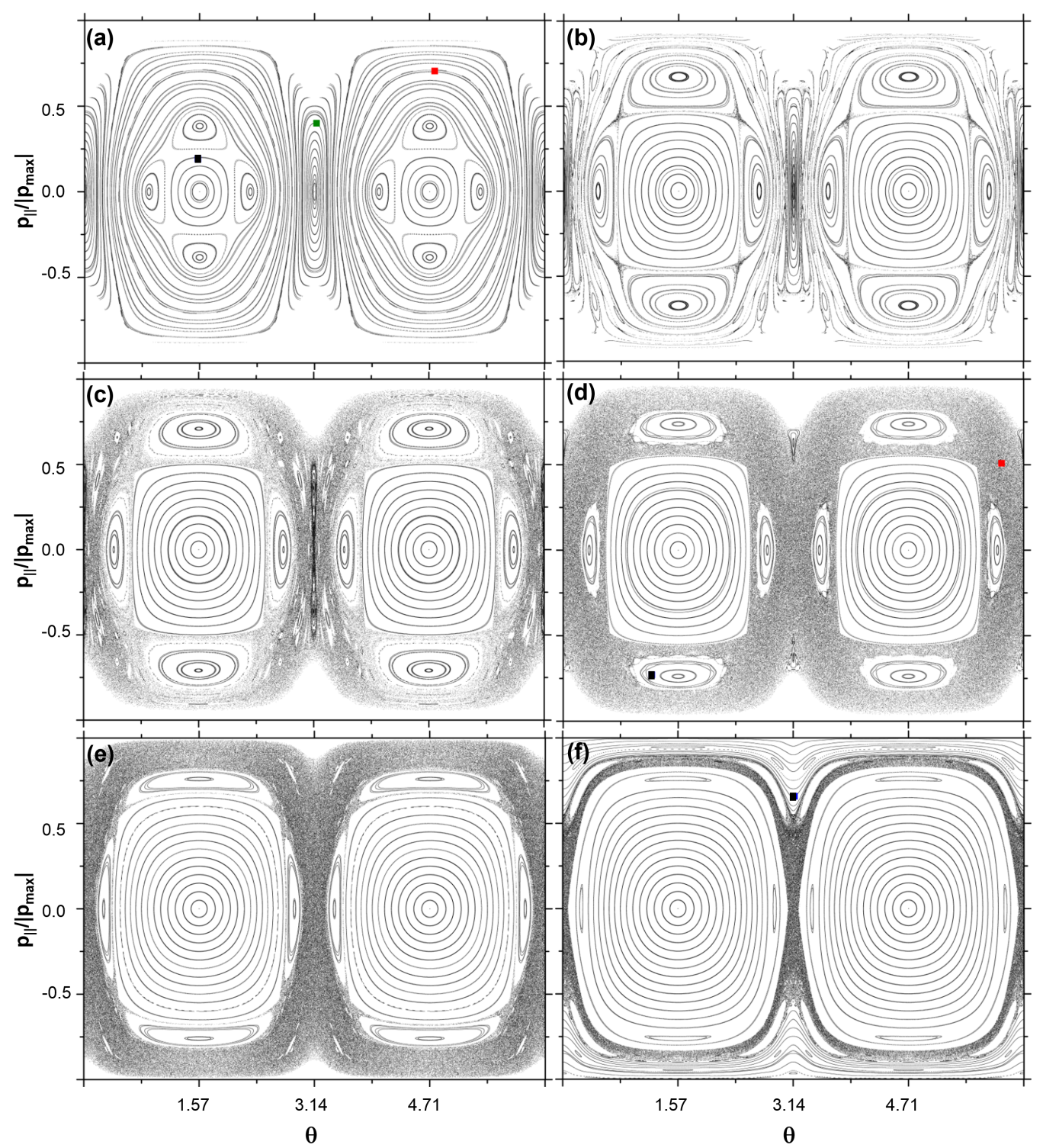

Figure 6: [Color on-line.] Same as in Fig. 5, but with eccentricity e $=0.84$. The small squares indicate initial conditions for the trajectories shown in Fig. 7.

\section{Parameter Space}

The Poincaré plots of Fig. 5 and 6 paint the following picture of the billiard dynamics, as the hardness $h$ is increased from zero: an initially reg- 

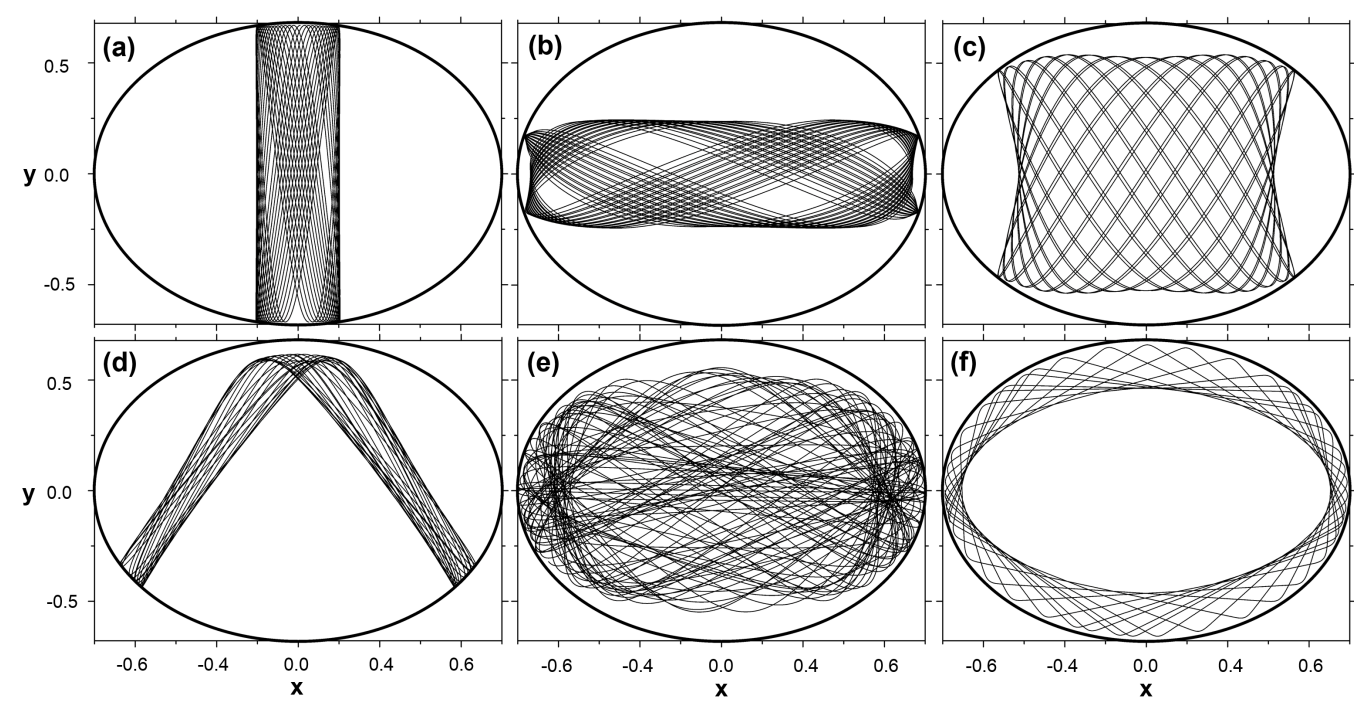

Figure 7: Trajectories corresponding to different regions of the phase space plots shown in Fig. 6, where the trajectories initial conditions are also indicated. Trajectories in (a), (b), and (c) start on the points in Fig. 6 (a) marked by the black, green and red squares; (d) and (e) correspond, respectively, to the black and red squares in Fig. 6 (d); and (f) corresponds to the initial condition marked in Fig. 6 (f).

ular dynamics experiences a break-up of integrability, chaoticity reaches a maximum for a specific value of $h$ followed by a gradual reduction of the chaotic sea in phase space, which asymptotically approaches to the hardwall limit $(h \rightarrow \infty)$ where the system becomes integrable again. Moreover, this scenario also depends on the geometric parameter $e$. In this section, we investigate the two-dimensional parameter space $(e, h)$ by means of the fraction of phase space area occupied by the chaotic sea.

The method used to estimate the occupation fraction is the box-counting analysis $[35,36]$. The phase space is divided in a grid and the fraction of boxes that contain at least one point of a given orbit is a good approximation of the area this orbit fills. Regular orbits describe points or curves in the Poincaré phase space and have zero area. Chaotic orbits, on the other hand, belong to a chaotic sea of finite area. We choose a $145 \times 145$ grid and evolve 51 initial conditions (one at the origin and the remaining randomly scattered) for $10^{4}$ reflections. The occupation fraction related to each initial condition are thus obtained. The largest value for these fractions is our estimative for 
the size of the chaotic sea. Performing this calculation for a grid of $(e, h)$ values, we obtain a detailed picture of the chaoticity of the billiard in its space of parameters.

We show in Fig. 8 (a) the space of parameters for orbits of energy $E=0$. The chaoticity of the system displays a clearly nonmonotonic dependence on both parameters. We consider $e>0.5$, since for lower eccentricities the system does not exhibit a relevant stochastic layer. Chaotic behavior is favored for hardness between the interval $2<h<5$ and eccentricity between the interval $0.8<e<0.9$, reaching the largest value $51 \%$ of phase space occupation for $(e, h) \approx(0.84,2.5)$. For hardness $h>2$ chaos is always present, but as $h$ is increased the chaotic sea can be less or more persistent depending on the eccentricity, being, for example, more fragile for $e=0.625$ and more robust for $e=0.9$. The fishbone-like fine structure is a result of high order resonances taking place, with the corresponding satellite islands becoming alternately larger and smaller as the parameters are varied.

The parameter space for orbits of energy $E=0.7$ is depicted in Fig. 8 (b). This is a level not far from the maximum energy for bound trajectories, where we have the condition $E<1$, and significantly higher than the $E=0$ orbits of Fig. 8 (a). Nonetheless, both plots are remarkably similar, suggesting that the energy of the particles trapped in the elliptical billiard is not a decisive parameter for the dynamics.
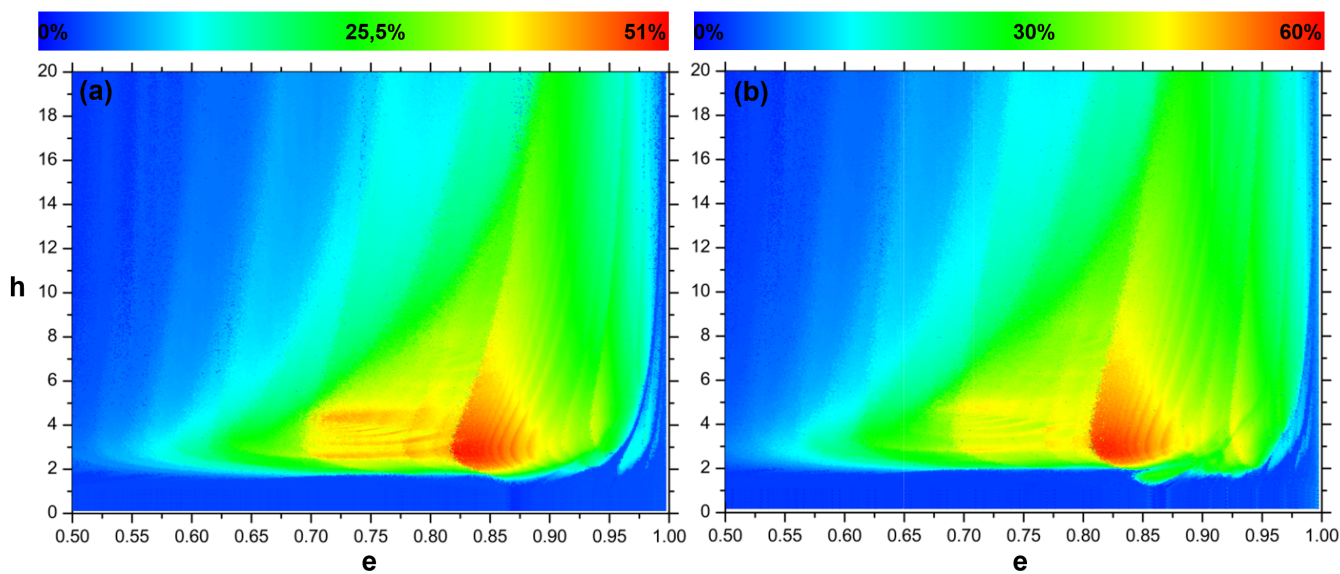

Figure 8: [Color on-line.] Parameter space for the soft-wall elliptical billiard. The color scale indicates the phase space fraction occupied by chaotic orbits of energy (a) $E=0$ and (b) $E=0.7$. 


\section{Conclusions}

In order to investigate the dynamical properties of the soft-wall elliptical billiard, we introduced a Hamiltonian model consisting of a smooth two-dimensional potential well that has the hard-wall elliptical billiard as a limiting case. We also defined a Poincaré surface of section using an analogue to the reflections with the hard walls, which allows a direct comparison of our results to the well-known properties of the hard-wall elliptical billiard.

We find that the soft-wall elliptical billiard, in contrast to its integrable hard-wall counterpart, presents chaos for wide ranges of eccentricity and hardness. This result complements the conclusions of Rom-Kedar et al. [19, 20, 21] and Kaplan et al. [22, 23, 24], who argue that the dynamics of hard-wall billiards turns more regular when softness (inverse of hardness) is incorporated into the system: The softness introduces resonances to the system and, while Rom-Kedar et al. show that these resonances lead to the appearance of islands in phase space which reduce the size of the chaotic sea, we have showed that these resonances can also destroy the heteroclinic structure that constitutes a separatrix, giving rise to a stochastic layer which can grow into a chaotic sea.

In the elliptical billiard, this stochastic layer expands as the potential becomes softer ( $h$ is reduced) and reaches a maximum. As we continue to reduce the hardness of the walls, the system recovers stability through a bifurcation cascade that culminates with the separatrix heteroclinic orbit at the origin, and its associated stochastic layer, being replaced by an island. We found the effect of $h$ on the chaotic sea to depend nontrivially on the eccentricity $e$ of the potential. The interplay of both quantities, $h$ and $e$, results in a rich parameter space structure which is still not fully explored.

\section{Acknowledgements}

This work was made possible through the financial support from the Brazilian research agencies CNPq and Fundação Araucária.

\section{References}

[1] N. Chernov, R. Markarian, Chaotic Billiards, American Mathematical Society: Mathematical Surveys and Monographs 127 (2006). 
[2] M. V. Berry, Statistics of nodal lines and points in chaotic quantum billiards: perimeter corrections, fluctuations, curvature, J. Phys. A: Math Gen. 35 (2002) 3025-3038.

[3] H. -J. Stöckmann, J. Stein, "Quantum" Chaos in Billiards Studied by Microwave Absorption, Phys. Rev. Lett. 64 (1990) 2215-2218.

[4] L. A. Ponomarenko, F. Schedin, M. I. Katsnelson, R. Yang, E. W. Hill, K. S. Novoselov, A. K. Geim, Chaotic Dirac Billiard in Graphene Quantum Dots, Science 18 (2008) 356-358.

[5] M. Hentschel, K. Ritchter, Quantum chaos in optical systems: The annular billiard, Phys. Rev. E 66 (2002) 056207-1-056207-13.

[6] O. Bohigas, M. J. Giannoni, C. Schmit, Phys. Rev. Lett. 52 (1984) 1-4.

[7] B. Dietz, A. Richter, Quantum and wave dynamical chaos in superconducting microwave billiards, Chaos 25 (2015) 097601-1-097601-17.

[8] S. K. Joseph, J. Sabuco, L. Y. Chew, M. A. F. Sanjuán, Entanglement Manipulation in a Quantum Chaotic Optical Fiber by Modifying its Geometry, Opt. Express (to be published).

[9] M. V. Berry, Regularity and chaos in classical mechanics, illustrated by three deformations of a circular "billiard", Eur. J. Phys. 2 (1981) 92-102.

[10] F. Lenz, F. K. Diakonos, P. Schmelcher, Classical dynamics of the timedependent elliptical billiard, Phys. Rev. E 76 (2007) 066213-1-066213-14.

[11] E. D. Leonel, L. A. Bunimovich, Suppressing Fermi Acceleration in a Driven Elliptical Billiard, Phys. Rev. Lett. 104 (2010) 224101-1-2241014.

[12] F. Lenz, F.K. Diakonos, P. Schmelcher, Tunable Fermi Acceleration in the Driven Elliptical Billiard, Phys. Rev. Lett. 100 (2008) 014103-1014103-4.

[13] F. Lenz, C. Petri, F. R. N. Koch, F.K. Diakonos, Evolutionary phase space in driven elliptical billiards, New J. Phys. 11 (2009) 083035-1083035-10. 
[14] D. F. M. Oliveira, M. Robnik, Scaling Invariance in a Time-Dependent Elliptical Billiard, Int. J. Bifurcat. Chaos 22 (2012) 1250207-1-12502077 .

[15] A. Loskutov, A. R. Ryabov, L. G. Akinshin, Properties of some chaotic billiards with time-dependent boundaries, J. Phys. A: Math. Gen. 33 (2000) 7973-7986.

[16] A. C. J. Luo, R. P. S. Han, The resonance theory for stochastic layers in nonlinear dynamic systems, Chaos, Solitons Fractals 12 (2001) 24932508 .

[17] S. S. Abdullaev, Canonical maps near separatrix in Hamiltonian systems, Phys. Rev. E 70 (2004) 046202-1-046202-20.

[18] Y-U Kim, U. Kuhl, H-J. Stöckmann, J. P. Bird, Investigating dynamical tunneling in open quantum dots by means of a soft-walled microwavecavity analogue, J. Phys.: Condens. Matter 17 (2005) L191-L198.

[19] V. Rom-Kedar, D. Turaev, Elliptic islands appearing in near-ergodic flows, Nonlin. 11 (1998) 575-600.

[20] V. Rom-Kedar, D. Turaev, Billiards: A singular perturbation limit of smooth Hamiltonian flows, Chaos 22 (2012) 026102-1-026102-21.

[21] V. Rom-Kedar, D. Turaev, Big islands in dispersing billiard-like potentials, Phys. D 130 (1999) 187-210.

[22] N. Friedman, A. Kaplan, D. Carasso, N. Davidson, Observation of Chaotic and Regular Dynamics in Atom-Optics Billiards, Phys. Rev. Lett. 86 (2001) 1518-1521.

[23] A. Kaplan, N. Friedman, M. Andersen, N. Davidson, Observation of Islands of Stability in Soft Wall Atom-Optics Billiards, Phys. Rev. Lett. 87 (2001) 274101-1-274101-4.

[24] A. Kaplan, N. Friedman, M. Andersen, N. Davidson, Stable regions and singular trajectories in chaotic soft-wall billiards, Phys. D, Nonlinear Phenom. 187 (2004) 136-145.

[25] R. Ketzmerick, Fractal conductance fluctuations in generic chaotic cavities, Phys. Rev. B 54 (1996) 10841-10844. 
[26] H. A. Oliveira, C. Manchein, M. W. Beims, Soft wall effects on interacting particles in billiards, Phys. Rev. E 78 (2008) 046208-1-046208-9.

[27] M. Hénon, C. Heiles, The applicability of the third integral of motion: Some numerical experiments, The Astrophys. J. 69 (1964) 73-79.

[28] G. Contopoulos, A third integral of motion in a galaxy, Z. Astrophys. 49 (1960) 273-291.

[29] B. Barbanis, On the Isolating Character of the "Third" Integral in a Resonance Case, The Astron. J. 71 (1966) 415-419.

[30] M. Tabor, Chaos and Integrability in Nonlinear Dynamics: An Introduction, Wiley, New York (1989).

[31] D. F. M. Oliveira, E. D. Leonel, On the dynamical properties of an elliptical-oval billiard with static boundary, Commun. Nonlinear Sci. Numer. Simulat. 15 (2010) 1092-1102.

[32] A. L. P. Livorati, A. Loskutov, E. D. Leonel, A family of stadium-like billiards with parabolic boundaries under scaling analysis, J. Phys. A: Math. Theor. 44 (2011) 175102-1-175102-12.

[33] P. Bálint, M. Halász, J. A. Hernández-Tahuilán, D. P. Sanders, Chaos and stability in a two-parameter family of convex billiard tables, Nonlin. 24 (2011) 1499-1521.

[34] J. Chen, L. Mohr, H.-K. Zhang, P. Zhang, Ergodicity of the generalized lemon billiards, Chaos 23 (2013) 043137-1-043137-12.

[35] H. R. Dullin, P. H. Richter, A. Wittek, A two-parameter study of the extent of chaos in a billiard system, Chaos 6 (1996) 43-58.

[36] V. Lopac, A. Šmić, Chaotic properties of the truncated elliptical billiards, Commun. Nonlinear Sci. Numer. Simullat. 16 (2011) 309-323. 